\title{
Aggregating partial, local evaluations to achieve global ranking
}

\author{
Paolo Laureti, Lionel Moret, Yi-Cheng Zhang \\ Department of Physics, University of Fribourg, Chemin du Muse 3, CH - 1700 \\ Fribourg, Switzerland
}

\begin{abstract}
We analyze some voting models mimicking online evaluation systems intended to reduce the information overload. The minimum number of operations needed for a system to be effective is analytically estimated. When herding effects are present, linear preferential attachment marks a transition between trustful and biased reputations.
\end{abstract}

Key words: Reputation systems, electronic communities, sorting.

\section{The General Problem}

Electronic communities of all kinds, transaction systems in particular, bring together users otherwise unknown to each other. In order for expressed opinions to be trusted or for transactions to materialize, rating systems are often crucial to rely upon $[1,2]$. They collect information about participants' past behavior, aggregate them and display the result. Such systems become most effective if posted ratings are weighted according to raters' reputation, the prototype application of this feedback technique being Epinions.

Previous work $[3,4,5]$ concentrates on optimal reputation systems, assuming that users pick the object to be judged from a uniform distribution and that the process converges to the real values, without bothering about the amount of resources spent. In reality we have to face a limited amount of evaluations and biased probability distributions of the number of votes.

Here we shall consider a system of $N$ objects endowed with an intrinsic quality $q_{i}, i=1,2, \ldots, N$. At each time step $t$, a user evaluates (or ranks) $k$ objects drawn from a probability distribution $p(t)$. We shall analyze different situations of increasing complexity within this framework, trying to find out under 
what circumstances the original values $\vec{q}=\left[q_{1}, q_{2}, \ldots, q_{N}\right]$ (or their ordering) can be reconstructed from the aggregation of the evaluations with non zero probability for $N \rightarrow \infty$.

\section{Random evaluations}

In this section we consider the case where objects to be evaluated are chosen from a uniform distribution $p_{i}(t)=1 / N$.

Occupancy problem. As a first example we fix $k=1$ and ask what is the minimum number of users $t_{1}$ needed to evaluate every object at least once with a given probability $P>0$. The probability that after $t$ steps all objects have been evaluated reads [6]:

$$
P(t, N)=\sum_{j=0}^{N}(-1)^{j}\left(\begin{array}{c}
N \\
j
\end{array}\right)\left(1-\frac{j}{N}\right)^{t} \underset{t, N \rightarrow \infty}{\sim} e^{-\lambda}
$$

where $\lambda \simeq N e^{-\frac{t}{N}}$ is the expected number of unjudged objects. The above limit becomes greater than zero as soon as the number of evaluators $t$ reaches the value

$$
t_{1} \simeq N \log \frac{N}{\lambda}
$$

Pairwise comparisons. Let us now fix $k=2$ and assume that players can just compare (not rate) one randomly chosen couple at each step. We call $r_{i}$ the $i^{\text {th }}$ ranked agent according to her intrinsic value $q_{i}$. If raters make no mistakes, the real rank is recovered once the $N-1$ paired comparisons $G_{N-1}=$ $\left[\left(r_{1}, r_{2}\right),\left(r_{2}, r_{3}\right), \ldots,\left(r_{N-1}, r_{N}\right)\right]$ have been performed. The probability to pick an element belonging to $G_{N-1}$ is $2 / N$, and from (1) one needs doing so at least $N \log N$ times. The minimal number of steps needed to find $\vec{r}=\left[r_{1}, r_{2}, \ldots, r_{N}\right]$ with finite probability is then

$$
t_{2}^{c} \simeq N^{2} \log (c N)
$$

where $c$ is a constant depending on $P$.

We should remark here that random comparisons are not at all a good solution for sorting a vector of scalars: finding optimal sorting algorithms is a classic problem of computer science [7]. In real situations, though, one does not always have control over the evaluators' choice. Besides, evaluators make mistakes and the outcome of a comparison is often uncertain. In this case a common procedure is to perform round robins with successive eliminations, obtaining equation (2) for the minimum number of comparisons needed to find the winner [8]. 
Ranking a group of peers. Let us now consider a self evaluating community, a group of $N$ users sharing the same expertise and voting for one another. This is equivalent to fixing $t=N$ in our general model. The $N$ agents pick each $k$ randomly chosen peers, and establish a partial ranking among them according to their intrinsic values. We want to find, in the limit of large $N$, the minimal average value $k_{r}$ of $k$ that allows to recover the "God-given" ordering with probability $P$.

When $k \ll N$ this model can be mapped into the previous one. In fact making a local ordering of $k$ elements would require about $k^{2}$ paired comparisons. We then need $N k_{r}^{2} \sim N^{2} \log N$ comparisons to find the entire ranking. Thus

$$
k_{r} \simeq \sqrt{N \log (c N)}
$$

In figure 1 this last result is shown to match well simulation data.

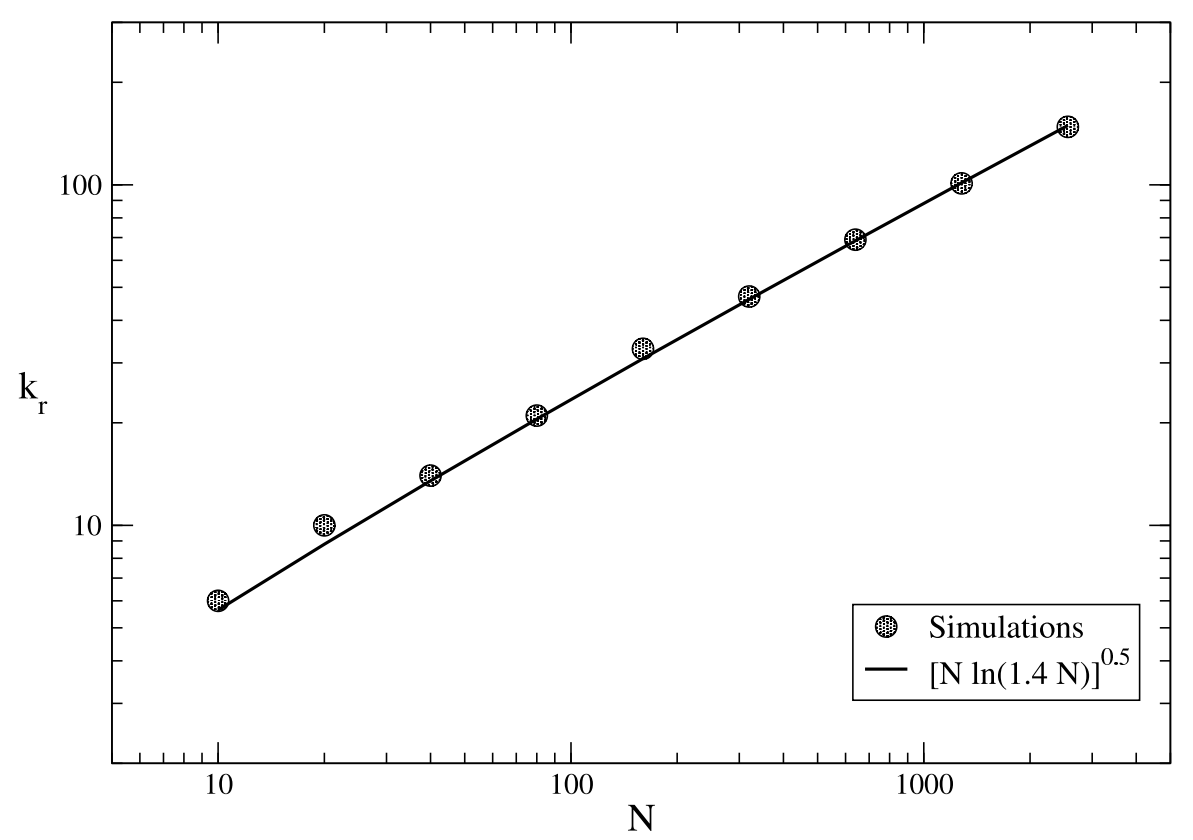

Fig. 1. Minimal average number of comparisons per agent $k_{r}$ needed to achieve the correct rank with probability $P=0.7$ as a function of $N$. Circles are simulation results averaged over 100 realizations, the solid line is the analytical estimation (3). 


\section{Preferential attachment}

In real electronic communities fame plays an important role. Objects that are already popular are more likely to receive comments, books mentioned by important media are more likely to be reviewed, people about whom many talk about are more likely to be judged again, and so on. Such "richer get richer" phenomenon, often referred to as preferential attachment [9], appears in numerous empirical data of social systems [10] and is thought to be one of the factors responsible for the emergence of scale free networks. A direct consequence of this mechanism is that attendance is not a fair indicator of web pages' quality [11].

Reputation systems collect evaluations about objects, aggregate and release them. The aggregation process often consists in computing an average of the received votes for each object. Assuming that objects have an intrinsic quality $q_{i}$ and that evaluations are random variables with mean equal to $q_{i}$, the law of large numbers ensures that computed reputations tend to the real intrinsic values once the number of users becomes large. The implicit assumption is that every object receives a growing number of evaluations, which is not always the case in the presence of some sort of preferential attachment.

This can be verified within the framework of the occupancy problem of section 2: a user per time step $t$ evaluates $k=1$ objects among the $N$ available. Instead of drawing the objects to be evaluated from a uniform distribution, let us now define

$$
p_{i}(t)=\frac{v_{i}^{\alpha}(t)}{\sum_{j=1}^{N} v_{j}^{\alpha}(t)},
$$

where $v_{i}(t)$ is the number of evaluations received by object $i$ up to time $t$, with $v_{i}(0)=1 \forall i$. The parameter $\alpha$ sets the strength of the preferential attachment. In the limit $\alpha \rightarrow \infty$ one has $p_{i}(t) \rightarrow \delta\left(i-i^{*}\right)$, where $\left.i^{*}=\operatorname{argmax}_{i}\left\{v_{i}(t)\right\}\right)$; if $\alpha=0$, on the other hand, $p_{i}$ becomes uniform.

As the real rank is not reached with certainty, it is useful to define a distance on the rank space. Let

$$
d(r[1], r[2])=\frac{1-c(r[1], r[2])}{2}
$$

be the distance between two ranks, where

$$
c(r[1], r[2])=\frac{\sum_{i=1}^{N} r_{i}[1] r_{i}[2]}{\sqrt{\sum_{i=1}^{N} r_{i}^{2}[1] \sum_{i=1}^{N} r_{i}^{2}[2]}}
$$

is the rank correlation coefficient [12]. 


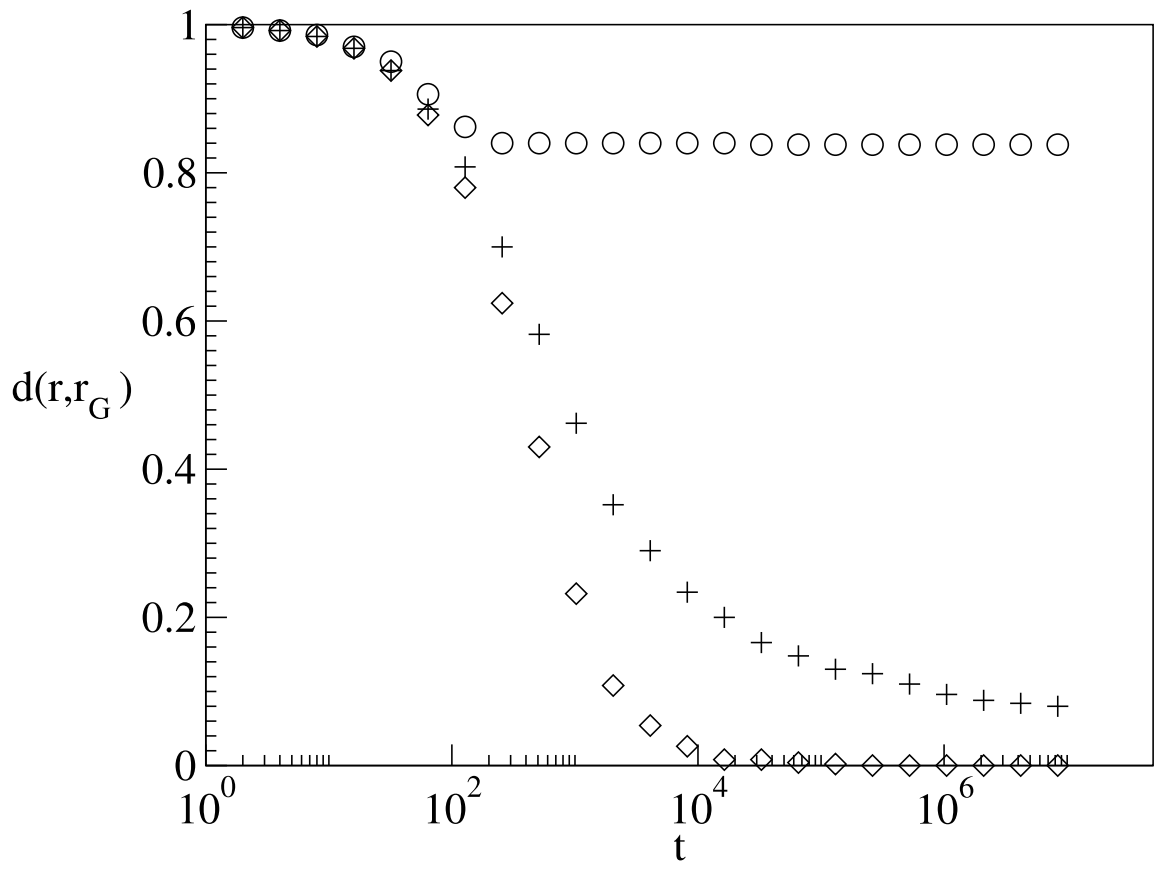

Fig. 2. Time dependence of the distance (5) between the "God given rank" $r_{G}$ and the rank $r$ produced by the model with preferential attachment (4). Symbols report, in log-linear scale, results of a simulation with $N=500$ agents and $\alpha=2$ (circles), $\alpha=1$ (plus signs) and $\alpha=0$ (diamonds).

In fig. 2 the time dependence of the distance between the "God given rank" and the best guess $r$, as defined in (5), is reported. Numerical simulations show that for $\alpha>1$ the distance reaches a plateau $>0$, meaning that one can never achieve an arbitrary good estimate of qualities $\vec{q}$ because some agents never get to be evaluated. For $\alpha<1$, on the other hand, the real rank is recovered in a finite number of time steps; at the transition point $\alpha=1$ one needs an infinite amount of steps to evaluate all objects.

In fact we can explain such a behavior with the following argument. The transition appears when the number of visits $v_{i}(t)$ does not grow in time for some $i$ s. For most objects the number of visits still grows linearly in time

$$
v_{i}(t) \simeq f_{i} t
$$

close to the transition. We can thus approximate the denominator of (4) $\sum_{j=1}^{N} v_{j}^{\alpha}(t) \simeq N t^{\alpha} I(\alpha)$, where $I(\alpha)=\frac{1}{N} \sum_{i=1}^{N} f_{i}^{\alpha}$. The probability for a given object $i$ never to receive an evaluation up to time $t$ is $Q_{i}(t)=\prod_{t^{\prime}=0}^{t}\left(1-p_{i}\left(t^{\prime}\right)\right)$, the $\log$ 
of which, $\log \left(Q_{i}\right) \simeq-\frac{1}{N I(\alpha)} \sum_{s=1}^{t} \frac{1}{s^{\alpha}}$, only converges to a finite value for $\alpha \geq 1$ : the transition value is indeed $\alpha=1$.

One can generalize the preferential attachment rule (4) by introducing multiplicative intrinsic qualities: high quality agents have more chance to be evaluated, similarly to the model proposed in ref. [13]. Let us define the probability to be evaluated as follows:

$$
p_{i}(t)=\frac{q_{i} v_{i}^{\alpha}(t)}{\sum_{j=1}^{N} q_{j} v_{j}^{\alpha}(t)}
$$

We can again approximate the denominator of $(7)$ as $\sum_{j=1}^{N} q_{j} v_{j}^{\alpha}(t) \simeq N t^{\alpha} I(\alpha)$, where $I(\alpha)=\int_{-\infty}^{+\infty} \rho(q) q f^{\alpha}(q) d q$ and $\rho$ is the probability distribution of the quality vector $q$. The same transition at $\alpha=1$ is found and equation (6) yields

$$
\begin{aligned}
f_{i} & =\left(\frac{q_{i}}{N I(\alpha)}\right)^{\gamma} \\
\gamma & =\frac{1}{1-\alpha} .
\end{aligned}
$$

Numerical simulations are shown to agree with this approximation in figure 3. The left panel displays the $q$ dependence of $f$ for fixed $\alpha$ : equation (8) asymptotically matches the simulation data. In the right panel of figure 3 the ratio $\gamma$ between the logarithm of the rate of visits $v_{i} / t$ and that of the intrinsic quality $q_{i}$ is plotted against $\alpha$. Equation (9) fits the data for $\alpha<\alpha_{c}$ and diverges at $\alpha=1$ as expected. The simulations were carried on with a uniform $\rho(q)$.

The distribution of the number of visits becomes a power law around the transition point $\alpha=1$, as reported in figure 4 .

The results described in this section can be easily extended to the case of pairwise comparisons introduced in section 2. That is, agents compare 2 objects per time step, chosen according to equation (7), and rank them in order of decreasing quality. The global ranking is finally established on the basis of these partial orderings. The question is to find the maximal value of $\alpha$ that allows to recover the true rank with finite probability. This can be done by evaluating the probability that two agents (belonging to the set $G_{N-1}$ ) will never be compared. By very similar reasoning to the absolute judgment case, one obtains $\alpha_{c}=\frac{1}{2}$. 

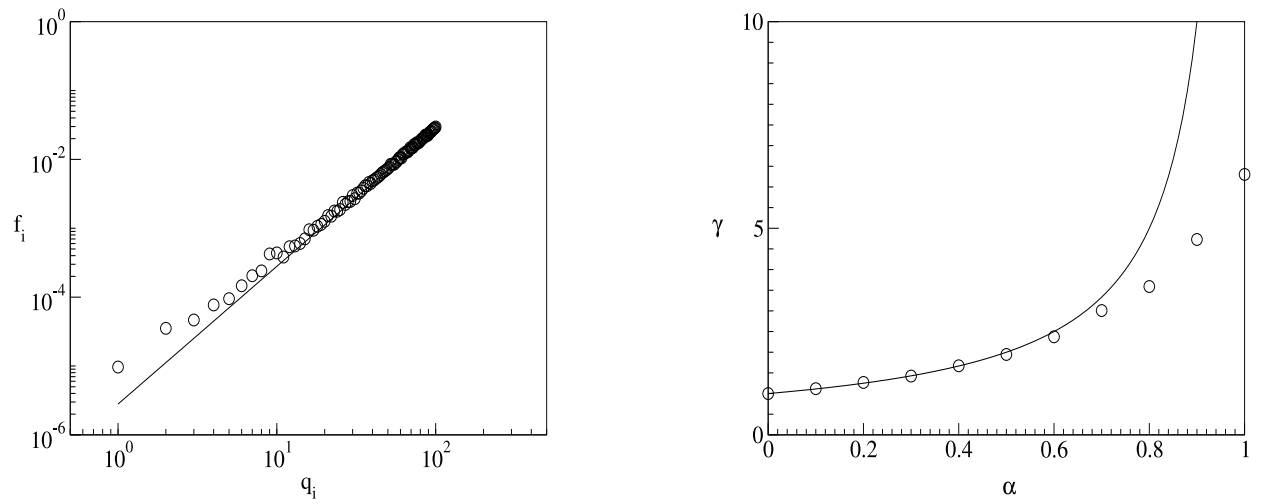

Fig. 3. (Left graph) Average rate of visits $f$ to an agent plotted against her intrinsic quality $q$, after $10^{5}$ time steps, with $\alpha=0.5$. (Right graph) Ratio $\gamma$ between the logarithm of the rate of visits $v_{i} / t$ and that of the intrinsic quality $q_{i}$ plotted against $\alpha$. Circles are simulation data, lines are calculated with equation 8 . Other simulatin parameters: number of agents $N=500$, number of realizations $=10$. Qualities are uniformly distributed.

\section{Aknowledgments}

For early collaboration on this paper and sharing insights we thank Hassan Masum and Yi-Kuo Yu; for discussions and a careful reading of the manuscript we thank Frantisek Slanina and Giancarlo Mosetti. This work was partially supported by the Swiss National Science Foundation through project number 2051-67733.

\section{References}

[1] H. Masum, Y.-C. Zhang, Manifesto for the reputation society, First Monday $9(7)$.

[2] P. Laureti, F. Slanina, Y.-K. Yu, Y.-C. Zhang, Buyer feedback as a filtering mechanism for reputable sellers, Physica A 316 (2002) 413-429.

[3] M. Chen, J. Singh, Computing and using reputations for internet ratings, in: Proceedings of the third ACM Conference on Electronic Commerce, ACM Press, New York, NY, USA, 2001, pp. 154-162.

[4] K. Fujimura, T. Nishihara, Reputation rating system based on past behavior of evaluators, in: Proceedings of the 4th ACM conference on Electronic commerce, ACM Press, 2003, pp. 246-247.

[5] G. Zacharia, A. Moukas, P. Maes, Collaborative reputation mechanisms in electronic marketplaces, in: Proceedings of the Thirty-second Annual Hawaii 


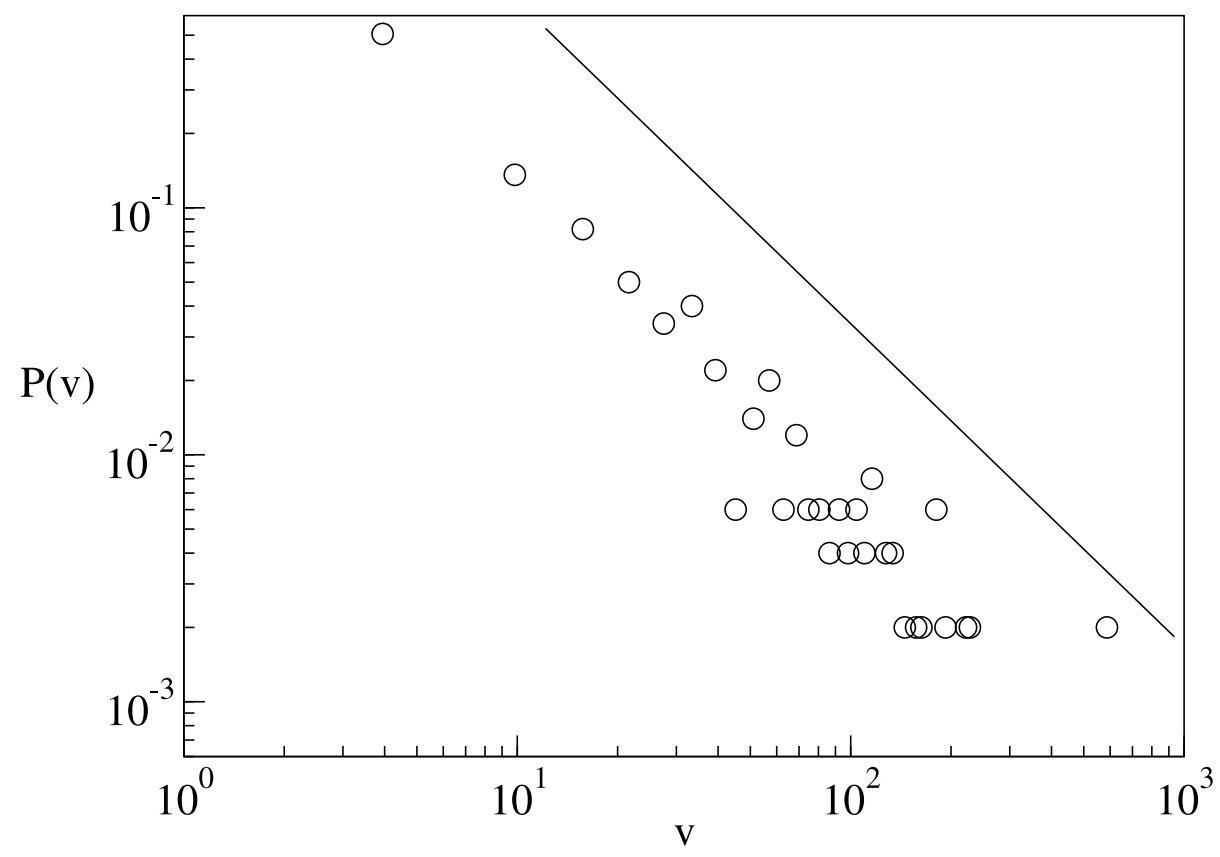

Fig. 4. Probability distribution $P(v)$ of the number of visits. Circles are simulation data of the model characterized by equation (7) with $\alpha=1$ and $N=500$, after $10^{5}$ steps. Here qualities are uniformly spaced, i.e. $q_{i}=i$. The solid line is a power law with exponent -1.3 .

International Conference on System Sciences-Volume 8, IEEE Computer Society, 1999, p. 8026.

[6] W. Feller, An introduction to probability theory and its applications, Vol 1, 3rd Edition, Wiley, 1968.

[7] D. E. Knuth, Sorting and Searching, Addison-Wesley, 1973.

[8] P. Laureti, J. Mathiesen, Y.-C. Zhang, Selection by pairwise comparisons with limited resources, Physica A 338 (2004) 596.

[9] R. Albert, A.-L. Barabási, Statistical mechanics of complex networks, Rev. Mod. Phys. 74 (2002) 47.

[10] R. Albert, A. Barabsi, H. Jeong, Scale-free characteristics of random networks: The topology of the world wide web (2000).

[11] G. Bianconi, P. Laureti, Y.-K. Yu, Y.-C. Zhang, Ecology of active and passive players and their impact on information selection, Physica A 332 (2004) 519532 .

[12] W. H. Press, B. P. Flannery, S. A. Teukolsky, W. T. Vetterling, Numerical 
Recipes: The Art of Scientific Computing, 2nd Edition, Cambridge University Press, Cambridge (UK) and New York, 1992.

[13] G. Bianconi, A.-L. Barabasi, Competition and multiscaling in evolving networks, Europhysics Letters 54 (2001) 436. 